\title{
Optimal switching using coherent control
}

\author{
Kristensen, Philip Trøst; Heuck, Mikkel; Mørk, Jesper
}

Published in:

Applied Physics Letters

Link to article, DOI:

10.1063/1.4789372

Publication date:

2013

Document Version

Publisher's PDF, also known as Version of record

Link back to DTU Orbit

Citation (APA):

Kristensen, P. T., Heuck, M., \& Mørk, J. (2013). Optimal switching using coherent control. Applied Physics Letters, 102(4), 041107. https://doi.org/10.1063/1.4789372

\section{General rights}

Copyright and moral rights for the publications made accessible in the public portal are retained by the authors and/or other copyright owners and it is a condition of accessing publications that users recognise and abide by the legal requirements associated with these rights.

- Users may download and print one copy of any publication from the public portal for the purpose of private study or research.

- You may not further distribute the material or use it for any profit-making activity or commercial gain

- You may freely distribute the URL identifying the publication in the public portal

If you believe that this document breaches copyright please contact us providing details, and we will remove access to the work immediately and investigate your claim 


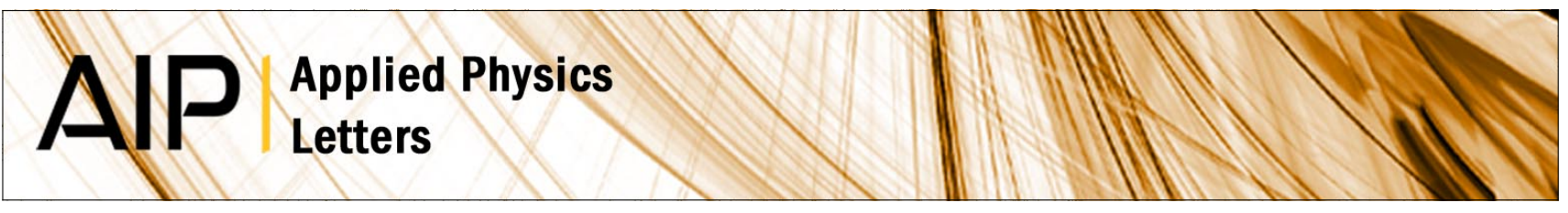

\section{Optimal switching using coherent control}

Philip Trøst Kristensen, Mikkel Heuck, and Jesper Mørk

Citation: Appl. Phys. Lett. 102, 041107 (2013); doi: 10.1063/1.4789372

View online: http://dx.doi.org/10.1063/1.4789372

View Table of Contents: http://apl.aip.org/resource/1/APPLAB/v102/i4

Published by the American Institute of Physics.

\section{Related Articles}

Bandstructure measurement in nonlinear optical waveguide arrays

Appl. Phys. Lett. 102, 081104 (2013)

Analysis of second-harmonic generation by primary horizontal shear modes in layered planar structures with imperfect interfaces
J. Appl. Phys. 113, 043513 (2013)

Optical bistability based on Bragg grating resonators in metal-insulator-metal plasmonic waveguides

AlP Advances 3, 012106 (2013)

Review Article: Quasi-phase-matching engineering of entangled photons

AIP Advances 2, 041401 (2012)

Dynamic nonlinear thermal optical effects in coupled ring resonators

AlP Advances 2, 032131 (2012)

\section{Additional information on Appl. Phys. Lett.}

Journal Homepage: http://apl.aip.org/

Journal Information: http://apl.aip.org/about/about_the_journal

Top downloads: http://apl.aip.org/features/most_downloaded

Information for Authors: http://apl.aip.org/authors

\section{ADVERTISEMENT}

\section{AIP Applied Physics Letters}

\section{EXPLORE WHAT'S NEW IN APL}

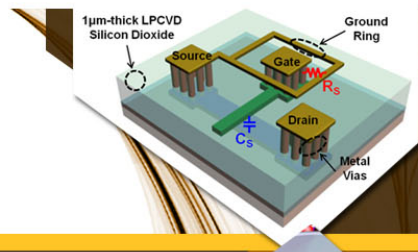

SURFACES AND INTERFACES

Focusing on physical, chemical, biological, structural, optical, magnetic and electrical properties of surfaces and interfaces, and more.. 


\title{
Optimal switching using coherent control
}

\author{
Philip Trøst Kristensen, Mikkel Heuck, and Jesper Mørk \\ DTU Fotonik, Technical University of Denmark, DK-2800 Kgs. Lyngby, Denmark
}

(Received 3 August 2012; accepted 10 January 2013; published online 28 January 2013)

\begin{abstract}
We introduce a general framework for the analysis of coherent control in coupled optical cavitywaveguide systems. Within this framework, we use an analytically solvable model, which is validated by independent numerical calculations, to investigate switching in a micro cavity and demonstrate that the switching time, in general, is not limited by the cavity lifetime. Therefore, the total energy required for switching is a more relevant figure of merit than the switching speed, and for a particular two-pulse switching scheme we use calculus of variations to optimize the switching in terms of input energy. (C) 2013 American Institute of Physics. [http://dx.doi.org/10.1063/1.4789372]
\end{abstract}

The high energy density in optical micro cavities may lead to pronounced non-linear material responses. ${ }^{1-3}$ This in turn provides a mechanism for time-domain all-optical switching in which a control pulse shifts the resonance of an optical cavity to regulate the transmission of a signal pulse. Design and operation of such switches have been based on different non-linear effects, including carrier dispersion induced by two-photon absorption in $\mathrm{GaAs}^{1}$ and both linear and two-photon absorption in InGaAsP. ${ }^{2}$ Central to any such cavity enhanced approach is the need for fast switching of the optical control energy in the cavity between the values corresponding to the "on" and "off" states of the switch, since residual energy may lead to unwanted patterning effects. ${ }^{4}$ The energy dynamics are intimately linked with the cavity quality factor, or $Q$-value, which sets the intrinsic time scale at which light enters and exits the (undriven) cavity. In particular, if the field in the cavity is excited by a short pulse, the field amplitude subsequently decays exponentially with a rate $\gamma=\omega_{\mathrm{C}} / 2 Q$, where $\omega_{\mathrm{C}}$ is the (angular) cavity resonance frequency. In addition to the amplitude, however, optical fields carry a phase which can be used for additional engineering of the coupling in and out of the cavity. This kind of coherent control is well known in the optical manipulation of atomic and molecular systems, ${ }^{5-7}$ where it is used to enhance transitions between specific atomic or molecular states while suppressing other (unwanted) transitions. Recently, coherent control was applied to improve the coupling of energy into an optical cavity, ${ }^{8}$ and in Ref. 9, two consecutive pulses with a $\pi$ phase difference were used to excite and deexcite the field in an optical cavity at timescales much faster than the cavity lifetime.

In this letter, we introduce a general framework for the analysis of coherent control in coupled cavity-waveguide systems and use it to find the optimal switching scheme. We first show that within the limits of our model, it is possible to switch the energy in the cavity arbitrarily fast. Next, we validate the model with independent numerical calculations and thus demonstrate clearly that the switching time, in general, is not limited by the cavity lifetime, consistent with Ref. 9. Therefore, from an optimization point of view, the total energy required for a given switching action is a more relevant figure of merit than the switching speed. Last, we solve the optimization problem analytically using calculus of variations and show that the optimal scheme is slightly different from our initial and more intuitive (constant power) approach. In this work, we focus on the energy dynamics of the control pulses. In practical realizations of cavityenhanced all-optical switching, the signal will typically be centered at a different optical frequency (possibly employing a different cavity resonance) and will have a lower power than the control. In such systems, the energy dynamics of the control pulses represent an inherent limiting factor for the performance. For a given energy threshold, our analysis provides a quite general and systematic approach for calculating the optimal operation of the control pulses. In particular, it directly allows us to calculate the generally time-dependent phase required for the fastest possible switching action, even in the non-linear case.

We consider a model system consisting of a cavity with a Kerr non-linearity coupled to two waveguides in an in-line configuration as illustrated in Fig. 1. We assume that the system is driven by an input field in the left waveguide of the form $S_{\text {in }}(t)=s_{\text {in }}(t) \exp \left(-\mathrm{i} \omega_{\mathrm{L}} t\right)$ and calculate the resulting fields in the cavity $A(t)=a(t) \exp \left(-\mathrm{i} \omega_{\mathrm{L}} t\right)$ and the right waveguide $S_{\text {out }}(t)=s_{\text {out }}(t) \exp \left(-\mathrm{i} \omega_{\mathrm{L}} t\right)$ using coupled mode theory (CMT). ${ }^{10}$ The slowly varying complex envelopes $a(t)$ and $s_{\text {out }}(t)$ solve the equations

$$
\begin{gathered}
\frac{\mathrm{d}}{\mathrm{d} t} a(t)=-\mathrm{i} \Delta \omega a(t)-\gamma a(t)+\sqrt{\gamma} s_{\text {in }}(t), \\
s_{\text {out }}(t)=\sqrt{\gamma} a(t),
\end{gathered}
$$

in which $\Delta \omega=\omega_{\mathrm{C}}-\omega_{\mathrm{L}}-F_{\mathrm{NL}}|a(t)|^{2}$, and $F_{\mathrm{NL}}$ is the effective Kerr coefficient that accounts for the material non-linearity as well as the field distribution in the cavity. ${ }^{11}$ The fields are normalized so that $P_{\text {in/out }}=\left|s_{\text {in/out }}(t)\right|^{2}$ and $U_{\mathrm{C}}=|a(t)|^{2}$ represent the instantaneous input/output power and the energy in the cavity, respectively. In order to analyze coherently

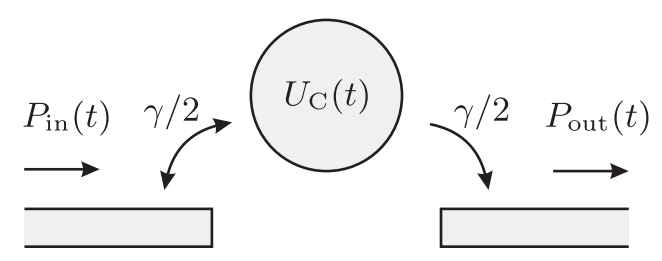

FIG. 1. Diagram of typical waveguide-cavity-waveguide system with input/ output powers $P_{\text {in/out }}(t)$ indicated along with the cavity energy $U_{\mathrm{C}}(t)$. 
controlled switching, we set $a(t)=|a(t)| \exp \{\mathrm{i} \varphi(t)\}$ and $s(t)=|s(t)| \exp \left\{\mathrm{i} \varphi_{\mathrm{S}}(t)\right\}$ and rewrite Eq. (1) as two real equations for the energy and the phase of the field in the cavity as

$$
\begin{gathered}
\frac{\mathrm{d}}{\mathrm{d} t} U_{\mathrm{C}}(t)=-2 \gamma U_{\mathrm{C}}(t)+2 \sqrt{U_{\mathrm{C}}(t) \gamma P_{\text {in }}(t)} \cos \{\Delta \varphi\}, \\
\frac{\mathrm{d}}{\mathrm{d} t} \varphi(t)=-\Delta \omega-\sqrt{\frac{\gamma P_{\text {in }}(t)}{U_{\mathrm{C}}(t)}} \sin \{\Delta \varphi\},
\end{gathered}
$$

where $\Delta \varphi=\varphi(t)-\varphi_{\mathrm{S}}(t)$, and we have assumed that $F_{\mathrm{NL}}$ is real (but non-zero, in general). From Eq. (3), the largest positive (negative) instantaneous rate of change of the cavity energy is found when $\Delta \varphi=p \pi$ and $p$ is even (odd). This explicitly shows how coherent control can improve the switching, and we note that given any $\varphi(t)$, one can set $\varphi_{\mathrm{S}}(t)=\varphi(t)-p \pi$. Therefore, we first solve Eqs. (3) and (4) assuming the phase relation to hold, and subsequently send in light with the correct phase. This procedure guarantees the fastest possible switching of the energy for a given $P_{\text {in }}(t)$. Incidentally, it also renders Eq. (3) independent of $\varphi(t)$, and one can solve this first and insert the solution in Eq. (4). This approach suggests a two-pulse scheme in which the first (second) pulse serves to increase (decrease) the cavity energy. We first consider a scheme in which the input power in each pulse is constant and we vary only the phase. We refer to this as the constant power approach. For switching on, we set $U_{\mathrm{C}}(0)=0$ and solve Eq. (3) with $P_{\text {in }}=P_{\text {on }}$ and $\Delta \varphi=0$ to find

$$
U_{\mathrm{C}}(t)=\frac{P_{\mathrm{on}}}{\gamma}\left(\mathrm{e}^{-\gamma t}-1\right)^{2} .
$$

The energy in the cavity thus increases, at a rate set by $\gamma$, towards the maximum energy of $P_{\text {on }} / \gamma$, as illustrated in Fig. 2(a). For any $t>0$ and any $U_{\mathrm{C}}(t)>0$, one can solve Eq. (5) to find the required input power $P_{\text {on }}$, illustrating explicitly
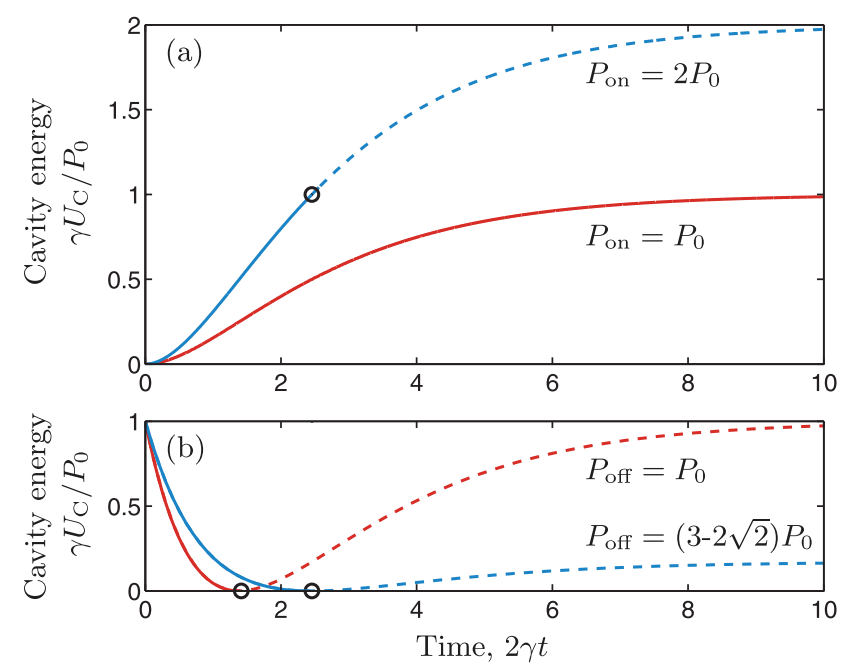

FIG. 2. Cavity energy variation $U_{\mathrm{C}}(t)$ in Eqs. (5) and (6), respectively, for switching up (a) and down (b) at different constant input powers $P_{\text {on }}$ and $P_{\text {off }}$, which in general can be controlled independently. Circle in (a) indicates the improvement in switching time for reaching $U_{\text {on }}=P_{0} / \gamma$ when doubling the input power from $P_{\text {on }}=P_{0}$ to $P_{\text {on }}=2 P_{0}$. Circles in (b) indicate the critical times at which the input power should be turned off in order to empty the energy from the cavity. Dashed lines indicate the cavity energy evolution if the input power is not turned off at the critical times. that the transition time can be made arbitrarily short. Similarly, for switching off, we set $U_{\mathrm{C}}(0)=U_{\text {on }}, P_{\text {in }}=P_{\text {off }}$, and $\Delta \varphi=\pi$ to find

$$
U_{\mathrm{C}}(t)=\frac{P_{\text {off }}}{\gamma}\left(\left(\sqrt{\frac{\gamma U_{\text {on }}}{P_{\text {off }}}}+1\right) \mathrm{e}^{-\gamma t}-1\right)^{2} .
$$

For the switch-off, interference effects act to decrease the energy in the cavity by coupling to the two waveguides at a rate that may be faster than the cavity decay rate. In this case, it is important to turn off the input driving field at the correct time lest the energy starts increasing again, as shown in Fig. 2(b). Equations (5) and (6) provide the cavity energy variation in the constant power approach. For linear problems at resonance $(\Delta \omega=0)$, the right hand side of Eq. (4) vanishes, leading to a piecewise constant phase, and we set $\varphi_{\mathrm{S}}^{\text {off }}=\varphi_{\mathrm{S}}^{\text {on }}+\pi$, where $\varphi_{\mathrm{S}}^{\text {on }}$ and $\varphi_{\mathrm{S}}^{\text {off }}$ represent the (constant) phases of the pulses used for switching on and off, respectively. In the general case, integration of Eq. (4) provides the required change in input phase.

The above analysis shows that within the validity of the model, there is no upper bound on the switching speed. The model is limited mainly by the single-mode assumption of the cavity which breaks down for very short pulses because of their large bandwidth. The example calculations below serve to validate the model for the choices of parameters in this letter. We consider a cavity in a finite sized onedimensional photonic crystal with lattice constant $\Lambda$ made from barriers of thickness $0.2 \Lambda$ and relative permittivity $\epsilon_{\mathrm{r}}=13$. As shown in Fig. 3, the cavity supports a cavity mode, $\tilde{f}_{\mathrm{C}}$, at the frequency $\tilde{\omega} \Lambda / 2 \pi c=0.2925-0.0009 \mathrm{i}$, where $\tilde{\omega}=\omega_{\mathrm{C}}-\mathrm{i} \gamma$, and $c$ is the speed of light. The (quasinormal) cavity mode ${ }^{12}$ was calculated as a solution to the Helmholtz equation with outgoing wave boundary conditions using a Fredholm-type integral equation ${ }^{13}$ and a one-dimensional version of the discretization in Ref. 14. This material system is immediately compatible with the CMT model in Eqs. (1) and (2). For reference, we use a time-dependent finite element (FEM) based model ${ }^{15}$ to provide completely independent calculations by direct solution of Maxwell's equations. From convergence studies, we estimate the maximum absolute error in the FEM calculations below to be less than $0.01 P_{0} / \gamma$. For simplicity as well as computational transparency, we have chosen

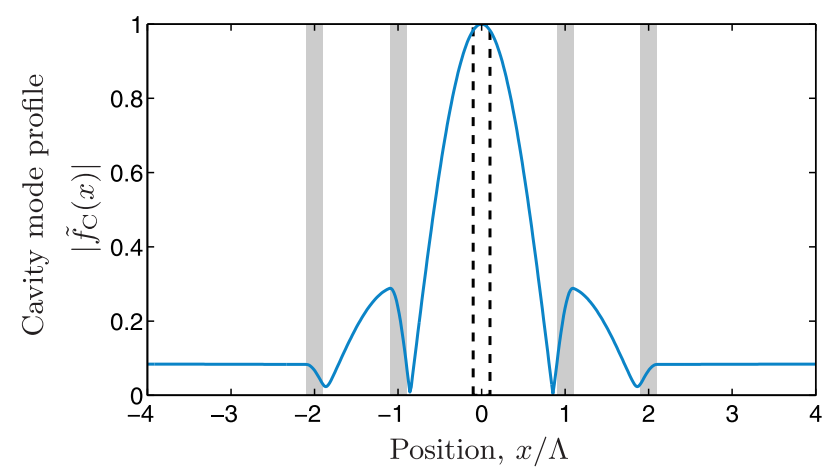

FIG. 3. Example cavity system in a finite one-dimensional photonic crystal with lattice constant $\Lambda$. Gray areas indicate the barriers and blue curve shows the absolute value of the cavity mode. Vertical dashed lines indicate the extent of non-linear material. 
a one-dimensional model system for validation of the CMT results. The CMT itself as well as the typical layouts of many technologically interesting systems, ${ }^{1,2}$ however, are effectively one-dimensional, and therefore the essential dynamics are fully captured by our model.

As an initial (linear) example, we set $F_{\mathrm{NL}}=0$ and calculate the energy dynamics in the model cavity when driven by the two-pulse scheme in the constant power approach. We choose a model switch-on/switch-off action with initial conditions given by $U_{\text {off }}=0$ and $U_{\text {on }}=P_{0} / \gamma$, respectively, and use two input pulses of width $T=1 / 2 \gamma$ with the second (switch-off) following directly after the first (switch-on). The required input powers are derived from Eqs. (5) and (6), and for this practical example we use super Gaussian pulses

$$
P_{\text {in }}=P_{\text {on/off }} \exp \left\{-\gamma\left(t-t_{0}\right)\right\}^{4 N},
$$

where $N=10$ and $t_{0}$ is the time offset. Fig. 4 shows the input pulses as well as the CMT solution and the direct FEM calculation of the time dependent cavity energy. We note that the finite value of the steepness parameter $N$ leads to a maximum energy which is slightly below target. Nevertheless, there is a very good agreement between the two solutions which clearly shows the validity of CMT for analyzing this kind of coupled waveguide-cavity systems. In the absence of the second input pulse, the energy leaks from the cavity at a rate $\Gamma=2 \gamma$ as shown by the dotted curve in the figure. Clearly, this is slower than in the case of coherent control using an "off" pulse. Overall, the analysis shows that the two-pulse scheme works as intended.

Next, for a non-linear example, we consider the model in Fig. 3 with an additional barrier at the center of the cavity. This barrier has a non-linear permittivity of the form $\Delta \epsilon_{\mathrm{NL}}$ $=\chi^{(3)} E(x, t)^{2}$ with $\chi^{(3)} P_{0} / c \epsilon_{0}=5 \times 10^{-4}$, where $E(x, t)$ is the electric field and $c$ and $\epsilon_{0}$ denote the speed of light and the permittivity of free space, respectively. Material nonlinearities are typically introduced in CMT using perturbation theory, and this has been shown to provide good agreement with full numerical calculations. ${ }^{8,10,16,17} \mathrm{We}$ note, however, that due to the finite $Q$-value of any real cavity the perturbation theory should be performed in the framework of non-Hermitian eigenvalue analysis ${ }^{12,18}$ as we do here. Although this seems to be largely ignored in the literature,

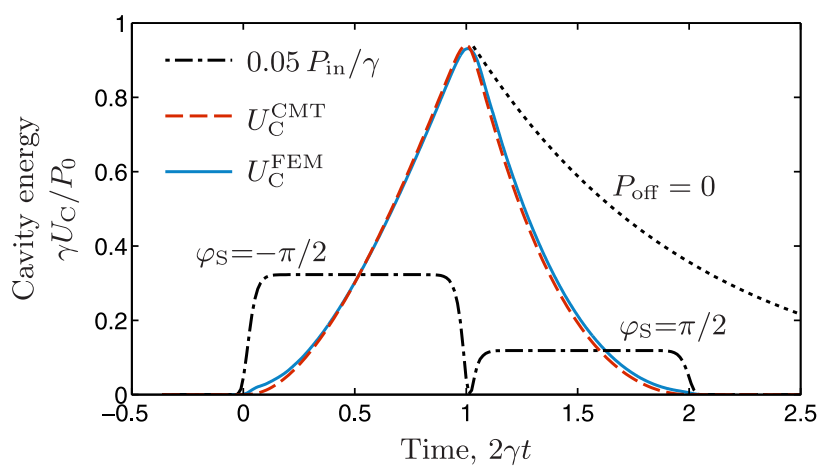

FIG. 4. Example of the switching scheme based on two input pulses (black dashed-dotted) with different phases as indicated. The CMT calculations (red dashed) compare well to independent FEM calculations (blue solid), confirming the applicability of the switching scheme. Dotted line shows the CMT solution in the absence of the second input pulse. we emphasize that the absolute value of the field in Fig. 3 is non-zero at all positions outside the cavity and diverges in the limit $x \rightarrow \pm \infty,{ }^{13}$ underscoring the need for a nonHermitian perturbation theory approach. Choosing the outermost edge of the photonic crystal as the boundaries of the cavity, we find $P_{0} F_{\mathrm{NL}} / \gamma^{2}=2.8+0.016$ i $^{11}$ Although $F_{\mathrm{NL}}$ is complex, the real part is two orders of magnitude larger than the imaginary part (which leads to a small change in decay rate), and neglecting the imaginary part leads to an absolute error of less than $5 \times 10^{-3} P_{0} / \gamma$ for the calculated energy dynamics below. This justifies our earlier assumption that $F_{\mathrm{NL}}$ is real and leads to the simple form for the energy dynamics in Eqs. (5) and (6). Fig. 5 shows results for both the piecewise constant input phase and a tailored input phase which takes the finite non-linear parameter into account. In both cases, we find a convincing correspondence between the two calculation methods, but only the tailored input phase results in the fast switch-off action of Eq. (6), clearly illustrating the importance of carefully adjusting the phase according to Eq. (4) with $\Delta \varphi=0$. The example calculations show the validity of the CMT and motivate direct use of the model for optimization.

Since (in the CMT) the switching can be made arbitrarily fast, the relevant quantity to optimize is not the switching time but rather the time-integrated input power required to perform the switching action in a certain time. Thus, for a given switching time $\Delta t=t_{2}-t_{1}$ and given initial and final cavity energies, $U_{\mathrm{C}}\left(t_{1}\right)$ and $U_{\mathrm{C}}\left(t_{2}\right)$, respectively, we seek the function $P_{\text {in }}(t)$ that minimizes the integral

$$
U_{\text {Switch }}=\int_{t_{1}}^{t_{2}} P_{\text {in }}(t) \mathrm{d} t,
$$

while maintaining the correct phase relation at all times. In practice, the boundary conditions $U_{\mathrm{C}}\left(t_{1}\right)$ and $U_{\mathrm{C}}\left(t_{2}\right)$ are given by the required energy in the cavity to switch between
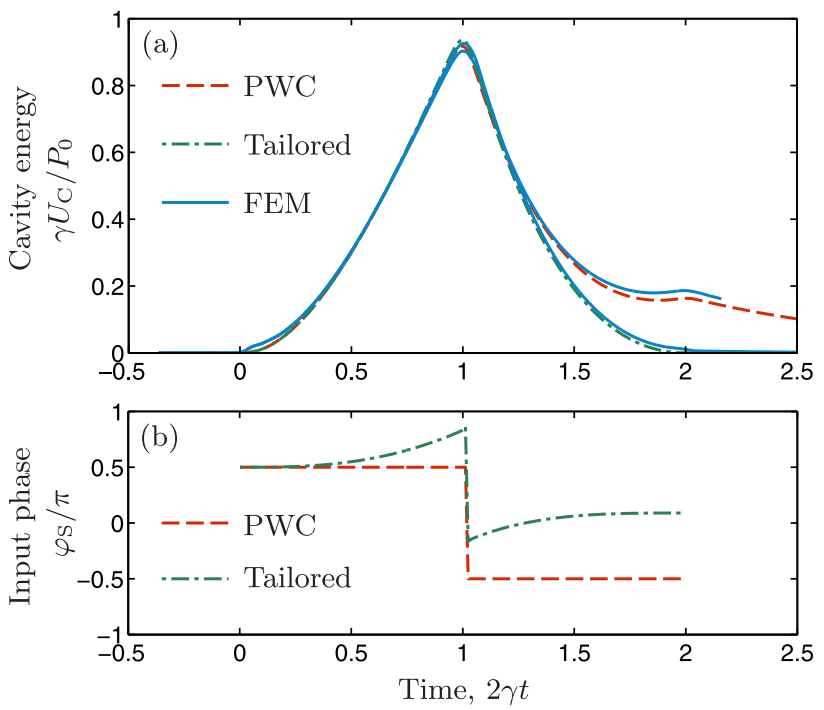

FIG. 5. Cavity energy dynamics (a) and input phase (b) for a two-pulse switching action in a cavity with a non-linear material response. Results are shown for both the cases of a piecewise constant (PWC) input phase (red dashed) and a tailored (green dashed-dotted) input phase. In both cases, independent FEM calculations of the cavity energy (blue solid) confirm the results. 


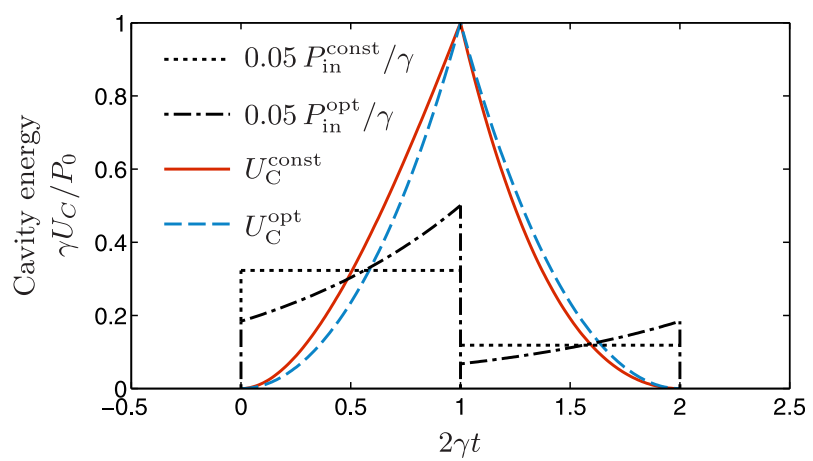

FIG. 6. Cavity energy variations in the optimal (blue dashed) and constant power (red full) schemes. Black dashed-dotted and dotted lines show the (scaled) input power variations.

the "on" and "off" states, as discussed in the introduction. From Eq. (3) with $\Delta \varphi=0$, we find that

$$
P_{\text {in }}(t)=\frac{1}{4 \gamma U_{\mathrm{C}}(t)}\left(\frac{\mathrm{d}}{\mathrm{d} t} U_{\mathrm{C}}(t)+2 \gamma U_{\mathrm{C}}(t)\right)^{2},
$$

and Eq. (8) takes the form of a classical optimization problem that we can solve using calculus of variations ${ }^{19}$ to find the optimum cavity energy variation $U_{\mathrm{C}}^{\mathrm{opt}}(t)$. For both the switch-on and the switch-off transitions, the optimum cavity energy variation is given as

$$
U_{\mathrm{C}}^{\mathrm{opt}}(t)=\frac{P_{0}}{2 \gamma}\left(\mathrm{e}^{2 \gamma t+c_{2}}+\frac{1}{4} c_{1}^{2} \mathrm{e}^{-2 \gamma t-c_{2}}-c_{1}\right),
$$

where the constants $c_{1}$ and $c_{2}$ are chosen to meet the appropriate boundary conditions. Equations (9) and (4) subsequently provide the appropriate input power and phase as a function of time. For the example switching problem, as previously stated the optimal cavity energy variation and input power variation are shown in Fig. 6. Within the validity of CMT, this represents the optimal way of switching the cavity energy via the two-pulse scheme. In the optimal approach, both the input power and the phase (not shown) vary nontrivially as a function of time. Nevertheless, comparing to the constant power scheme we find a relatively small difference in integrated power (areas under the black dasheddotted and dotted curves in Fig. 6) of approximately 2\%, which shows that for this particular choice of material system and switching requirements the constant power approach is close to optimal.

In conclusion, we have analyzed a two-pulse scheme for fast switching by coherent control. The applicability of the switching scheme as well as the underlying model has been validated by direct numerical evaluation of the wave equation, and we found a convincing agreement between the analytical and numerical results. Based on the model, we have subsequently analyzed the two-pulse approach using calculus of variations to find the optimal switching scheme. In this work, we have focused on Kerr non-linearities in which an intuitive constant power approach is close to optimal. For other types of non-linear interactions, a suitably modified version of the CMT provides a convenient equation system for similar optimizations, although this may not be analytically solvable in general. For a particular material system, the optimal solution may serve as an important tool in assessing the performance of a given switching scheme.

This work was supported by the EU FP7 project "Copernicus," the Villum Kann Rasmussen Center of Excellence NATEC and the Danish Council for Independent Research (FTP 10-093651).

${ }^{1}$ C. Husko, A. De Rossi, S. Combrie, Q. V. Tran, F. Raineri, and C. W. Wong, Appl. Phys. Lett. 94, 021111 (2009).

${ }^{2}$ K. Nozaki, T. Tanabe, A. Shinya, S. Matsuo, T. Sato, H. Taniyama, and M. Notomi, Nature Photon. 4, 477 (2010).

${ }^{3}$ M. Soljačić, M. Ibanescu, S. G. Johnson, Y. Fink, and J. D. Joannopoulos, Phys. Rev. E 66, 055601 (2002).

${ }^{4}$ M. Heuck, P. T. Kristensen, and J. Mørk, Opt. Express 19, 18410 (2011).

${ }^{5}$ W. S. Warren, H. Rabitz, and M. Dahleh, Science 259, 1581 (1993).

${ }^{6}$ N. Dudovich, D. Oron, and Y. Silberberg, Phys. Rev. Lett. 88, 123004 (2002).

${ }^{7}$ H. Rabitz, New J. Phys. 11, 105030 (2009).

${ }^{8}$ S. Sandhu, M. L. Povinelli, and S. Fan, Appl. Phys. Lett. 96, 231108 (2010).

${ }^{9}$ M. Ferrera, Y. Park, L. Razzari, B. E. Little, S. T. Chu, R. Morandotti, D. J. Moss, and J. Azaña, Nat. Commun. 1, 29 (2010).

${ }^{10}$ J. D. Joannopoulos, S. G. Johnson, J. N. Winn, and R. D. Meade, Photonic Crystals-Molding the Flow of Light, 2nd ed. (Princeton University Press, 2008).

${ }^{11}$ M. Heuck, P. T. Kristensen, and J. Mørk, in Proceedings of CLEO/QELS (2012), p. JW4A.6.

${ }^{12}$ H. M. Lai, P. T. Leung, K. Young, P. W. Barber, and S. C. Hill, Phys. Rev. A 41, 5187 (1990).

${ }^{13}$ P. T. Kristensen, C. Van Vlack, and S. Hughes, Opt. Lett. 37, 1649 (2012).

${ }^{14}$ P. T. Kristensen, P. Lodahl, and J. Mørk, J. Opt. Soc. Am. B 27, 228 (2010).

${ }^{15}$ We used Comsol multiphysics 4.2.

${ }^{16}$ M. F. Yanik, S. Fan, M. Soljačić, and J. D. Joannopoulos, Opt. Lett. 28, 2506 (2003).

${ }^{17}$ J. Bravo-Abad, S. Fan, S. G. Johnson, J. Joannopoulos, and M. Soljačić, J. Lightwave Technol. 25, 2539 (2007).

${ }^{18}$ K. M. Lee, P. T. Leung, and K. M. Pang, J. Opt. Soc. Am. B 16, 1418 (1999).

${ }^{19}$ B. Van Brunt, The Calculus of Variations (Springer, 2004). 